\title{
POLYNILPOTENT GROUPS OF PRIME EXPONENT
}

\author{
BY N. D. GUPTA
}

Communicated by W. Feit, November 17, 1967

Let $\gamma_{n}(G)$ denote the $n$th term of the lower central series of a group $G$ and define $\gamma_{m} \gamma_{n}(G)=\gamma_{m}\left(\gamma_{n}(G)\right)$. For a fixed positive integer $k$ define

$$
f_{k}(1)=1 \text { and } f_{k}(n)=f_{k}[n / 2]+k f_{k}[(n+1) / 2]
$$

for all $n>1$. In this paper we prove

THEOREM. Let $\left(m_{1}, \cdots, m_{t}\right)$ be a finite sequence of positive integers exceeding 1 and let $G$ be a group of prime exponent $p$ ( $p$ odd). Then

where

$$
\gamma_{r_{t}}(G) \subseteq \gamma_{m_{1}} \gamma_{m_{2}} \cdots \gamma_{m_{t}}(G),
$$

$$
r_{t}=m_{t}+\sum_{i=1}^{t-1}\left(m_{i}-1\right) f_{p-2}\left(m_{i+1}\right) \cdots f_{p-2}\left(m_{t}\right) .
$$

If $m_{1}=m_{2}=\cdots=m_{t}=2, r_{t}=1+\sum_{t=0}^{t-1}(p-1)^{i}$, a result of Tobin [2]. In general we have

$$
\gamma_{2} \gamma_{2} \cdots \gamma_{2}(G) \subseteq \gamma_{m_{1}} \gamma_{m_{2}} \cdots \gamma_{m_{t}}(G) \quad\left(\gamma_{2} \text { appears } u_{t} \text { times }\right)
$$

where $u_{t}=k+\sum_{j=1}^{t-1}\left(m_{j}-1\right)$ and $k$ is the least positive integer satisfying $2^{k} \geqq m_{t}$; so that the theorem of Tobin yields

$$
\gamma_{s_{t}}(G) \subseteq \gamma_{m_{1}} \gamma_{m_{2}} \cdots \gamma_{m_{t}}(G),
$$

where $s_{t}=1+\sum_{i=0}^{u_{t}-1}(p-1)^{i}$. The bound $r_{t}$ is in general far less than the known bound $s_{t}$. For instance in the very special case $\left(m_{1}, m_{2}, \cdots, m_{t}\right)=\left(2,2^{2}, \cdots, 2^{t}\right)$ while $r_{t}<s_{t}$ we further observe that the degree of the polynomial $r_{t}$ in $p$ is $\left(t^{2}+t-2\right) / 2$ as compared with $2^{t}-2$ in $s_{t}$.

The proof of the theorem is shown to follow from the following

Lemma. ${ }^{1}$ Let $G$ be a group of prime exponent $p$ ( $p$ odd) and let $N$, $A, B$ be subgroups of $G$ such that $N$ is normal in $G$ and $B \subseteq A$. Then $(N, A, B, \cdots, B) \subseteq(N,(A, B))(N, N)$ (B appears $p-2$ times).

With $N=G^{\prime}$ and $A=B=G$, one gets the well-known MeierWunderli's result that metabelian groups of prime exponent $p$ are nilpotent of class at most $p$. Since

$$
\left(\gamma_{[n / 2]}(G), \gamma_{[(n+1) / 2]}(G)\right) \subseteq \gamma_{n}(G) \text { and } \gamma_{[(n+1) / 2]}(G) \subseteq \gamma_{[n / 2]}(G),
$$

\footnotetext{
${ }^{1}$ For notation and other undefined terms the reader is referred to M. Hall [1].
} 
we get

$$
\begin{aligned}
&\left(N, \gamma_{[n / 2]}(G), \gamma_{[(n+1) / 2]}(G), \cdots, \gamma_{[(n+1) / 2]}(G)\right) \subseteq\left(N, \gamma_{n}(G)\right)(N, N) \\
&\left(\gamma_{[(n+1) / 2]}(G) \text { appears } p-2 \text { times }\right) ;
\end{aligned}
$$

and hence (by repeated applications)

$$
(N, G, \cdots, G) \subseteq\left(N, \gamma_{n}(G)\right)(N, N) \quad\left(G \text { appears } f_{p-2}(n) \text { times }\right) .
$$

By repeated applications of the lemma with suitable choices of $N$, $A, B$ it is now routine to compute that

$$
\gamma_{r_{t}}(G) \subseteq \gamma_{m_{1}} \gamma_{m_{2}} \cdots \gamma_{m_{t}}(G) \text {, where } r_{t} \text { is as defined before. }
$$

Proof of the Lemma. Put $(N, N)=\{1\}$ and $(N,(A, B))=\{1\}$ for $d \in N$ and $\alpha_{1}, \alpha_{2}, \cdots, \alpha_{l}$ in tegers, define

$$
d^{\sum_{i=1}^{l} \alpha_{i} x_{i}}=\prod_{i=1}^{l}\left(d^{\left.\alpha_{i}\right)^{x_{i}}}\right.
$$

for all $x_{1}, \cdots, x_{l}$ in $G$ (here $\left.y^{x}=x^{-1} y x\right)$. It is easily verified that

$$
d^{\alpha_{1} x+\alpha_{2} y}=d^{\alpha_{2} y+\alpha_{1} x} \text { and } d^{x-1}=d^{-1+x}=(d, x)
$$

for all $d \in N$ and $x, y \in G$; and $d^{a b}=d^{b a}$ for all $d \in N, a \in A, b \in B$. Further it is easily seen that the proof of the Lemma consists in showing that $\left(d, a, b_{2}, \cdots, b_{p-1}\right)=1$ or equivalently

$$
d^{(a-1)\left(b_{q}-1\right) \cdots\left(b_{p-1-1}\right)}=1
$$

for all $d \in N, a \in A, b_{2}, \cdots, b_{p-1} \in B$.

Since $1=\left(d x^{-1}\right)^{p}=d d^{x} \cdots d^{x^{p-1}}$, we have

$$
d^{1+x+\cdots+x^{p-1}}=1
$$

for all $d \in N$ and $x \in G$, which give in turn

$$
\begin{aligned}
& d^{\left(1+a b+\cdots+a^{p-1} b^{p-1}\right)-\left(1+b+\cdots+b^{p-1}\right)}=1 ; \\
& d^{b(a-1)+b^{2}\left(a^{2}-1\right)+\cdots+b^{p-1}\left(a^{p-1}-1\right)}=1 ; \\
& d^{(a-1)+b\left(a^{2}-1\right)+\cdots+b^{p-2}\left(a^{p-1}-1\right)}=1,
\end{aligned}
$$

for all $d \in N, a \in A, b \in B$.

We shall prove by induction on $j \in\{0, \cdots, p-2\}$, that

$$
d^{p i=0} b^{i-1} b^{i}\left(a^{i+j+1}-1\right)\left(b_{2}^{i+1}-1\right) \cdots\left(b_{j+1}^{i+1}-1\right)=1,
$$


for all $d \in N, a \in A, b, b_{2}, \cdots, b_{j+1} \in B$. For $j=0$, the result comes from (1). Suppose (2) holds for some $j \in\{0, \cdots, p-3\}$. In (2) replacing $b$ by $b b_{j+2}$ and taking quotient with (2) gives

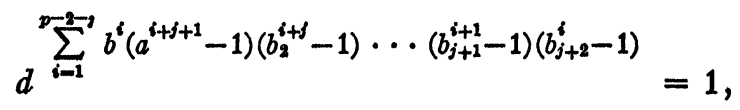

which is the same as

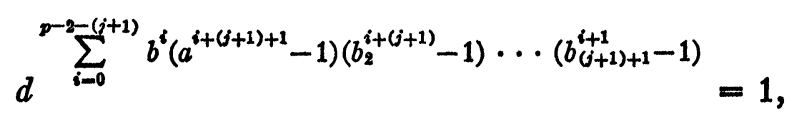

and (2) is proved for all $j \in\{0, \cdots, p-2\}$. Taking $j=p-2$ in (2) gives

$$
d^{\left(a^{p-1}-1\right)\left(b_{2}^{p-2}-1\right) \cdots\left(b_{p-1}-1\right)}=1
$$

for all $d \in N, a \in A$ and $b_{2}, \cdots, b_{p-1}$ in $B$. Replacing $a, b_{2}, \cdots, b_{p-2}$ by their suitable powers we get the required result.

Remark. The following result of Professor N. S. Mendelsohn (verbal communication) is of independent interest and provides an easy recognition of the function $f_{k}(n)$. If $n=2^{\alpha_{1}}+2^{\alpha_{2}}+\cdots+2^{\alpha_{m}}$, where $\alpha_{1}>\alpha_{2}>\cdots>\alpha_{m} \geqq 0$, then

$$
f_{k}(n)=(1+k)^{\alpha_{1}}+\sum_{i=2}^{m}(1+k)^{\alpha_{i}} k^{\alpha-1-\alpha_{i}-i+3} .
$$

Notice that the largest power of $k$ occurring in $f_{k}(n)$ is $\alpha_{1}+1$.

\section{REFERENCES}

1. Marshall Hall, Jr., The theory of groups, Macmillan, New York, 1959. 270.

2. Séan Tobin, On a theorem of Baer and Higman, Canad. J. Math 8 (1956), 263-

UNIVERSITY OF MANITOBA 